\title{
Time delays in the gravitationally lensed quasar SDSS J1004+4112
}

\author{
Janine Fohlmeister* \\ Heidelberg University \\ E-mail: janineeari.uni-heidelberg.de
}

The wide separation gravitational lens SDSS J1004+4112 is a rare example of a quasar lensed by an intervening galaxy cluster. We present four years of optical monitoring data for the four brightest images observed between December 2003 and June 2007. We measure the time delays, finding longer values than previously predicted. The time delay between the A and B images is $\Delta \tau_{B A}=40.6 \pm 1.8$ days in the expected sense that $\mathrm{B}$ leads $\mathrm{A}$ and the overall time ordering is C-B-A-D-E. We find that image $\mathrm{C}$ leads image $\mathrm{A}$ by $\Delta \tau_{C A}=821.6 \pm 2.1$ days. The lower limit on the remaining delay is that image $\mathrm{D}$ lags image $\mathrm{A}$ by $\Delta \tau_{A D}>1250$ days. With a delay of 2.3 years between the images $\mathrm{A}$ and $\mathrm{C}$, we even measure the longest time delay known in any gravitationally lensed quasar. Image $\mathrm{C}$ being the leading one, its light curve gives a 2.3 year forecast on the expected variations in the A/B image pair, that will allow for detailed remonitoring of sharp flux changes and for applying reverberation mapping techniques. The long delays allow us to fill in the seasonal gaps and assemble a continuous, densely sampled light curve spanning 5.7 years whose variability implies a structure function with a logarithmic slope of $\beta=0.52 \pm 0.02$. After correcting for the time delays, the residuals of the light curves clearly indicate that microlensing is present. Based on the microlensing of images $\mathrm{A}$ and $\mathrm{B}$, we estimate that the accretion disk size of the quasar source at a rest wavelength of $2300 \AA$ is $10^{14.8 \pm 0.3} \mathrm{~cm}$ for a disk inclination of $\cos i=1 / 2$.

The Manchester Microlensing Conference: The 12th International Conference and ANGLES Microlensing Workshop

January 21-25, 2008

Manchester, $U K$

\footnotetext{
* Speaker.
} 


\section{Introduction}

The wide-separation lensed quasar SDSS J1004+4112 was discovered in the Sloan Digital Sky Survey search for lenses (Inada et al. 2003; Oguri et al. 2004). The quasar at $z_{s}=1.734$ is split into five images with a maximum image separation of 14."62. It is one of the rare examples of a quasar gravitationally lensed by a cluster at $z_{l}=0.68$ (Inada et al. 2003, Wambsganss et al. 2003). Additional multiply imaged arcs formed from still higher redshift background galaxies have been detected (Sharon et al. 2005) and there is strong evidence for a faint fifth lensed image of the quasar located near the center of the brightest cluster galaxy (Inada et al. 2005).

Several theoretical studies of the time delays in SDSS J1004+4112 (Oguri et al. 2004; Williams \& Saha 2004; Kawano \& Oguri 2006) have explored their dependence on the mean mass profile of the cluster, finding a broad range of potential delays. The models indicate that the delay between the A and B images is relatively short (weeks) and that its value should indicate the magnitude of the much longer (years) delays between the $\mathrm{C}$ and $\mathrm{D}$ images. Time delay measurements in lensed quasars can be used to determine the Hubble constant independently of local distance estimators (Refsdal 1964, Oguri et al. 2007) or to study the structure of the lensing cluster. Time delay estimates are furthermore required to distinguish intrinsic source variability from variations due to microlensing by stars in the lensing galaxies.

\section{Data and Observations}

The photometric monitoring observations presented here took place between December 2003 and June 2007. Table 1 gives a summary of the involved telescopes and number of epochs (one epoch being one observing night). The bulk of these data were taken with the $1.2 \mathrm{~m}$ telescope at Fred Lawrence Whipple Observatory on Mount Hopkins (Arizona). The combined data set consists of 533 epochs. The average sampling rate is once every third day. In each observing night at least three 300s exposures were taken. The images of each night were then combined to improve the signal-to-noise for the further analysis. Regions around each of the quasar images and five standard stars were fitted to determine the relative fluxes and the structure of the PSF.

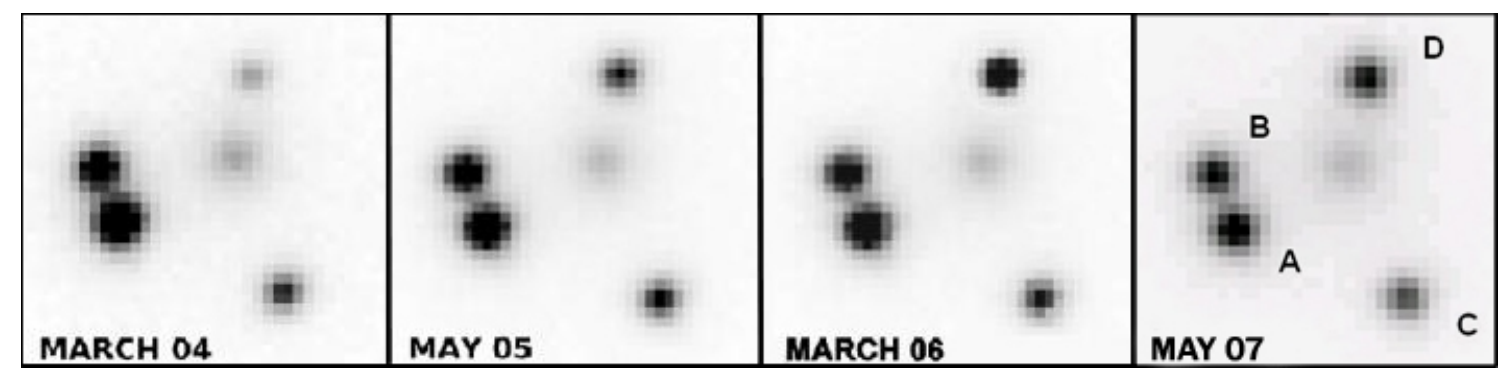

Figure 1: The panels show $23^{\prime \prime} \times 23^{\prime \prime}$ insets on the four bright quasar images at four observing epochs separated by about one year. The faint source in their middle is the bright galaxy belonging to the lensing cluster.

Figure 2 shows the resulting light curves of the 4 bright quasar components. All four bright quasar images show variability of more than 0.15 mag within each observing season. The small panels in Figure 1 show snapshots of the four bright quasar images at four different observing 
Table 1: Summary of Monitoring Observations

\begin{tabular}{lcccc}
\hline \hline Telescope & Detector & Pixel Scale ["/pixel] & $N_{\text {epoch }}$ & Filter \\
\hline FLWO 1.2m & 4Shooter & 0.666 & 93 & $\mathrm{R}$ \\
& Minicam & 0.604 & 74 & $\mathrm{r}$ \\
& Keplercam & 0.672 & $4+178$ & $\mathrm{R}+\mathrm{r}$ \\
APO 3.5m & SPICam & 0.282 & 9 & $\mathrm{r}$ \\
MDM 2.4m & Retrocam & 0.259 & 47 & $\mathrm{r}$ \\
& 8K & 0.344 & 12 & $\mathrm{R}$ \\
& Templeton & 0.508 & 6 & $\mathrm{R}$ \\
MDM 1.3m & Echelle & 0.275 & 3 & $\mathrm{R}$ \\
Palomar 1.5m & Templeton & 0.508 & 8 & $\mathrm{R}$ \\
Wise 1.0m & SITe & 0.379 & 13 & $\mathrm{R}$ \\
& Tektronix & 0.696 & 30 & $\mathrm{R}$ \\
WIYN 3.5m & TAVAS & 0.991 & 53 & clear \\
\hline
\end{tabular}

epochs. These images illustrate how images A and B slowly faded during the course of the four seasons while image D became significantly brighter. During the first season image D was very faint and almost not detectable in the background level of the frames. Over the whole four seasons image $\mathrm{D}$ brightened by more than $1.5 \mathrm{mag}$ and became the second brightest of the four images. For the purposes of measuring the B-A time delay, the most interesting features are the minima in the B light curve near days 3150, 3750 and 4190 in the first, third and fourth seasons respectively, and the corresponding features in the A light curve roughly 40 days later.

The galaxies of the lensing cluster are not detectable in the individual observations, except for the bright galaxy close to image D (G1 in Oguri et al. 2004). The fifth quasar image, E, is also too faint to be detected in our frames. Its position is near the center of the bright galaxy G1.

\section{Time Delay Measurements}

Many techniques for calculating time delays from light curves of lensed quasar images have been established. Here we apply three that produce mutually consistent results.

The simplest approach is the $\chi^{2}$-minimization, where the observed light curves $A\left(t_{i}\right)$ and $B\left(t_{i}\right)$ are cross-correlated with linearly interpolated light curves $a(t)$ and $b(t)$ for the other image. Assuming the time series $A\left(t_{i}\right)$ and $B\left(t_{i}\right)$ are undisturbed replicas of the same underlying original quasar light curve shifted in time, the difference of both $A\left(t_{i}\right)-b\left(t_{i}-\tau\right)$ for the correct time delay $\tau$ should be a constant $m(\tau)$ and reflect the magnification ratio of the two images.

As our second method for the determination of the time delay between images A and B we applied the dispersion spectra method developed by Pelt et al. $(1994,1996)$. For this no interpolation between the data points to compare the light curves of the two images is needed. Instead, a combined light curve is constructed by shifting the data points of one image in magnitude and time and combining them with the data points of the other image. 


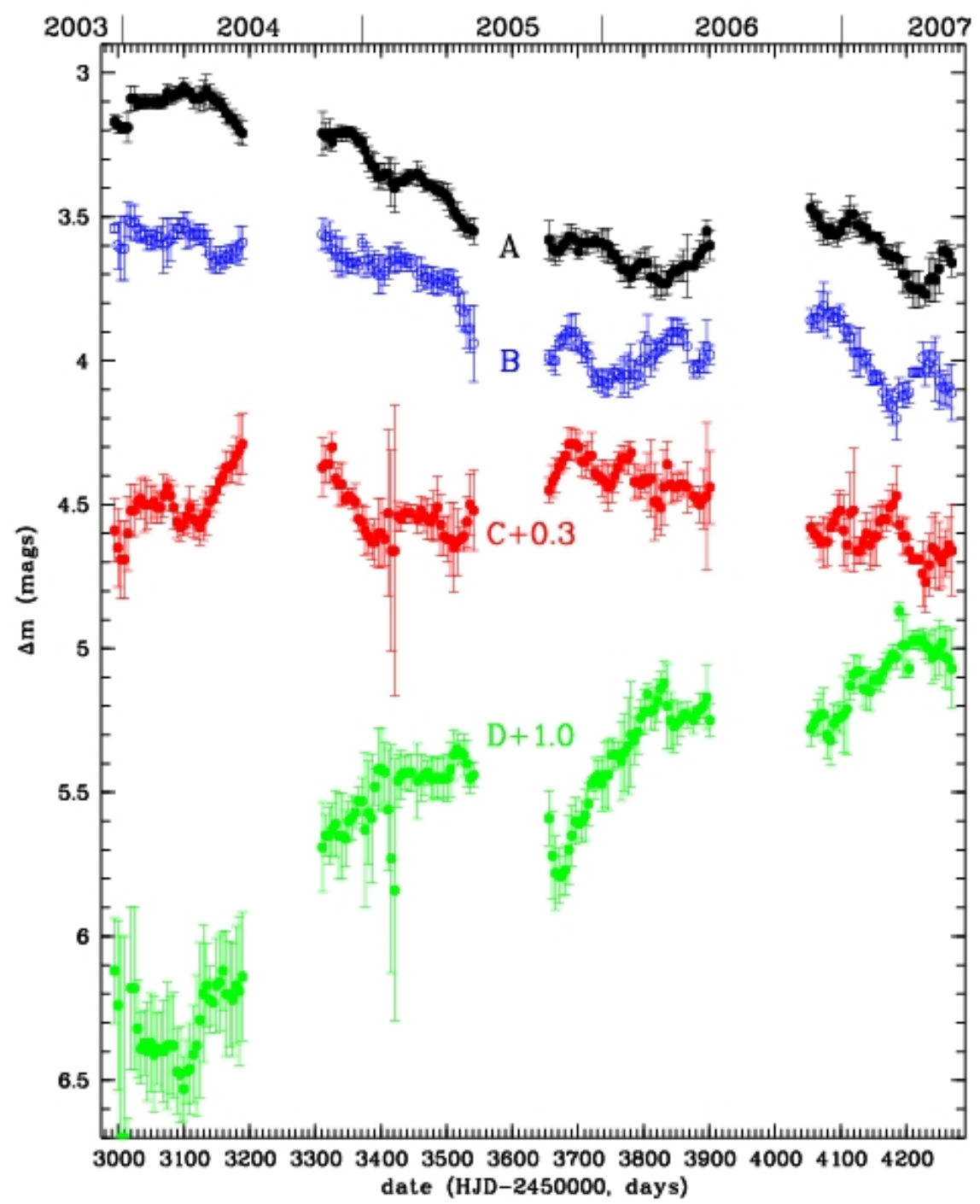

Figure 2: Light curves of the A, B, C and D images of SDSS J1004+4112 from December 2003 to June 2007. We present a running average of the data (one point every five days averaged over \pm 7 days) to emphasize the trends rather than the noise.

Both the $\chi^{2}$ and minimum dispersion methods treat the flux ratios between the images within each season as a constant. Either method could be modified to allow for more complex microlensing variations, but for our final analysis we use the polynomial fitting method of Kochanek et al. (2006), since it can most easily incorporate the effects of microlensing on both the delays and their uncertainties. In the polynomial method, each light curve is decomposed into intrinsic and extrinsic variations, in order to estimate the differential time delays between the images and to analyze the microlensing variability. The analysis of the combined data yields a delay between image A and $\mathrm{B}$ of $\Delta t_{B A}=40.6 \pm 1.8$ days.

For the more widely separated $\mathrm{C}$ and $\mathrm{D}$ images theoretical models predicted time delays of the order of months to years (Oguri et al. 2004; Williams \& Saha 2004; Kawano \& Oguri 2006). After four years of monitoring the time range covered by the light curves provided sufficient overlap to 
measure the time delays for the wide separated (14."62) image $C$ relative to the close image pair. The very characteristic features in the light curves of the images A and B in the third and fourth season and of image $\mathrm{C}$ in the first and second season are suggestive for detecting a time delay "by eye". The bump at the beginning of the first season in $\mathrm{C}$ is very similar to that observed at the end of the third season in image $\mathrm{B}$. There is a decline of about 0.3 mag at the beginning of the second season in image $\mathrm{C}$ that is repeated in image $\mathrm{B}$ in the fourth season, followed by a clear minimum.

For the statistical analysis of the time delay the methods described above were applied. Using the dispersion spectra method (Pelt et al. 1994, 1996), we find $\Delta \tau_{C A}=822 \pm 7$ days. To probe the result independently of the image A light curve, we check the time shift between the light curves of image $\mathrm{C}$ and $\mathrm{B}$. The time ordering of the images being C-B-A-D, we expect a value of $\Delta \tau_{C B}=\Delta \tau_{C A}-\Delta \tau_{B A}=782 \pm 7$ days. Our analysis yields a value of $\Delta \tau_{C B}=780 \pm 6$ days, which is consistent with the prediction. The measurement for the time delay between images $\mathrm{C}$ and $\mathrm{A}$ is slightly less accurate than the time delay between $\mathrm{C}$ and $\mathrm{B}$, because the shifted $\mathrm{A}$ light curve has less overlap with the image $\mathrm{C}$ light curve due to the seasonal gaps. For the polynomial method analysis we simultaneously fit A, B and C holding the B-A delay fixed to 40.6 days and find a C-A delay of $821.6 \pm 2.1$ days. With a value of $821.6 \pm 2.1$ days (2.3 years), the C-A delay is the longest gravitational lens time delay measured so far, almost doubling the previous longest value of Q $0956+561$. With image $C$ leading images A and B by 2.3 years, sharp variations in image $C$ can be used to plan intensive monitoring of images A and B.

The observed light curve of image $\mathrm{D}$ is mainly rising during the overall course of our monitoring, while the light curve of image A is mainly fading. From the derived ordering of the images, we know that image D should lag the other three images. No feature is seen in the light curve of image $\mathrm{D}$ that can be matched to the first season of image A. From this behavior we can derive a lower limit on the time delay between the images $\mathrm{A}$ and $\mathrm{D}$ of $\Delta \tau_{D A}>1250$ days (3.4 years).

\section{Microlensing}

Figure 3 shows the superposition of the shifted A, B and C light curves and the differences between them. From the residuals we can estimate the differential microlensing variability compared to image B. Image B was arbitrarily assumed to represent the intrinsic variability of the source quasar, because its light curve has most overlap with the other two. Figure 3 clearly shows that the residuals of the $\mathrm{A}$ and $\mathrm{C}$ light curves indicate that microlensing with amplitudes of order $0.15 \mathrm{mag}$ is present. We modeled the microlensing for images $\mathrm{A} / \mathrm{B}$ using the Bayesian Monte Carlo method of Kochanek (2004). We used the microlensing parameters of our lens model, with convergence $\kappa$ and shear $\gamma$ values of $\kappa=0.48$ and $\gamma=0.57$ for $\mathrm{A}$ and $\kappa=0.47$ and $\gamma=0.39$ for B. We find that the accretion disk size at a rest wavelength of $2300 \AA$ is

$$
R_{2300}=10^{14.8 \pm 0.3} \frac{\mathrm{cm}}{h_{70} \sqrt{\operatorname{cosi}}}
$$

for a disk inclination angle $i$, independent of whether we use a prior on the mean microlens mass of $0.1 M_{\odot}<\langle M\rangle<M_{\odot}$ or not. 


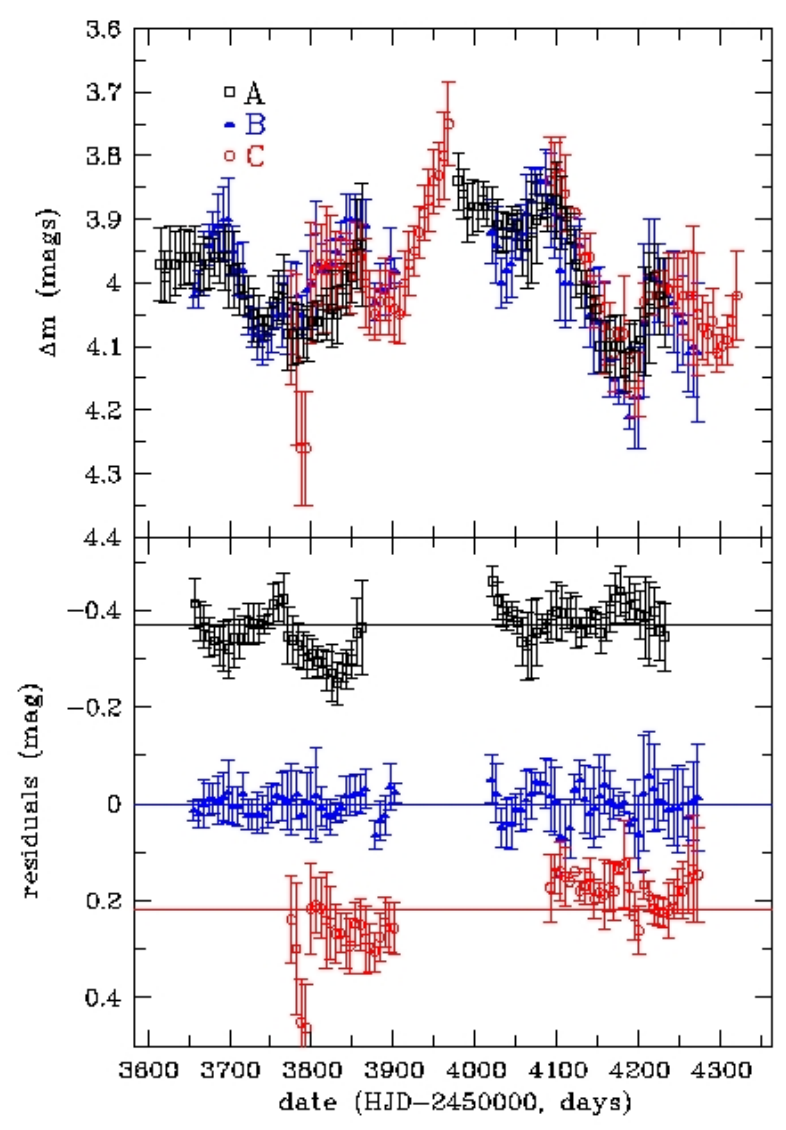

Figure 3: The image A, B and C light curves of SDSS J1004+4112 in their overlap region after shifting by the respective time delays. The data are binned in one week intervals with error bars derived by error propagation from the measurement errors. The lower box shows the residual magnitudes shifted by the offset between the images, revealing microlensing variability of order $0.15 \mathrm{mag}$. The light curve of image B was chosen to have constant flux because it has the most overlap with the other two images.

\section{The Structure Function}

One way to probe the time scales and amplitudes of quasar variability is the construction of the structure function. The quasar structure function measures the variability in the magnitude as a function of time lag between observational epochs. It is defined as

$$
S(\Delta t)=\sqrt{\frac{1}{N(\Delta t)} \sum_{i<j}\left[\left(m\left(t_{j}\right)-m\left(t_{i}\right)\right)^{2}-\sigma_{j}^{2}-\sigma_{i}^{2}\right]},
$$

where $\left(m\left(t_{j}\right)-m\left(t_{i}\right)\right)$ is the magnitude difference between two observations that are offset by a time lag $\Delta t$ and $m\left(t_{j}\right)$ is the measured magnitude with uncertainty $\sigma_{j}$ at epoch $t_{j}$. The sum runs over $N(\Delta t)$ epochs for which $\Delta t=t_{j}-t_{i}$, and only includes those time differences where data is available. The structure function can be fitted as a single power law

$$
S(\Delta t)=S_{100}\left(\frac{\Delta t}{100 \text { days }}\right)^{\beta}
$$


where $\Delta t$ is the rest-frame time difference and $S_{100}$ is the amplitude for a time lag of 100 days.

For SDSS J1004+4112 we can determine the structure function over a moderate time range and with a dense sampling rate and no seasonal gaps if we use the time-delay connected quasar light curves. Such data generally do not exist; structure functions for individual quasars have been determined with very sparse observational sampling of only a few epochs per year (e.g. Hawkins 2007) or in the composite form for big quasar samples but also very sparse sampling of about two epochs per quasar (Vanden Berk et al. 2004, de Vries et al. 2005, Wilhite et al. 2008).

We compute the structure function for the time-delay connected B and C light curves. These cover a time-baseline of 2065 days (5.7 years) in the observer's frame, corresponding to a maximum rest-frame baseline at $z_{s}=1.734$ of 755 days. For the BC light curves we also computed the structure function subtracting the microlensing variability estimate found in the time delay analysis. For the very different behavior of the image D light curve, which could not yet be timedelay connected to the other images, we compute the structure function independently for rest frame time lags up to 470 days. The slopes of the structure function are mutually consistent, with $\beta_{B C}=0.52 \pm 0.02$ and $\beta_{B C^{\prime}}=0.54 \pm 0.02$ after subtracting the estimated microlensing variability. Microlensing has little effect on the results because the source variability $(\sim 0.7 \mathrm{mag})$ greatly exceeds the microlensing variability $(\sim 0.15 \mathrm{mag})$. After a rest frame time lag between two observations of 100 days ( 9 months in the observers frame), the mean magnitude difference for the BC light curve is $S(\Delta t=100)=0.19 \pm 0.05 \mathrm{mag}$. For image $\mathrm{D}$ we find a similar albeit steeper slope of $\beta_{D}=0.55 \pm 0.03$, as expected from the light curve. The mean magnitude difference after 100 days, $S(\Delta t=100)$, is $0.30 \pm 0.06$ mag and about 0.1 magnitude higher than that determined from the BC light curve. For the composite structure functions of large quasar samples, the average amplitudes at 100 days were $S_{100} \simeq 0.11$ (Vanden Berk et al. 2004), 0.23 (de Vries et al. 2005) and 0.121 mag (Wilhite et al. 2008). The structure function of SDSS J1004+4112 is steeper and shows a higher amplitude than the average quasar. The observed higher variability amplitude of the fainter image D could be related to the anticorrelation of variability with quasar luminosity found in large surveys of quasar variability.

Theoretical models of quasar variability involving a starburst scenario predict slopes of $0.74 \leq$ $\beta \leq 0.90$, whereas accretion disk instability models predict shallower slopes of $0.41 \leq \beta \leq 0.49$ (Kawaguchi et al. 1998), as well as a flattening of the structure function after $\sim 100$ days in both cases. Our measurements are in disagreement with those predictions.

\section{Conclusions}

We have presented four seasons of monitoring data for the four bright images of the five image gravitational lens system SDSS J1004+4112. By using three different methods we measured the time delay between the merging A and B image pair, finding that B leads A by $40.6 \pm 1.8$ days. Together with model predictions for the arrival times, this fixes the overall time ordering of the images to be C-B-A-D-E. We also measured the very long delay for image $\mathrm{C}$, finding that it leads image A by $821.6 \pm 2.1$ days ( 2.3 years). We note that this is nearly twice the longest previously measured delay (the 417 day delay in Q 0957+561, Schild \& Thomson 1995, Kundic et al. 1997). We find a lower bound that $\mathrm{D}$ lags A by more than 1250 days (3.4 years). 
We have also clearly detected microlensing variability in the B-A images, with changes of order $0.15 \mathrm{mag}$ in the B-A flux ratio over the course of the four observing seasons. Based on the microlensing light curve, we estimate that the accretion disk size of the quasar source at a rest wavelength of $2300 \AA$ is $10^{14.8 \pm 0.3} \mathrm{~cm}$ for a disk inclination of $\cos i=1 / 2$.

The ability to construct a continuous light curve of the intrinsic variability and to use image $\mathrm{C}$ to provide early warning of sharp flux changes that can then be intensively monitored in images A and B make this system a good candidate for applying reverberation mapping techniques to a massive, luminous quasar. At present, the structure function of this system indicates that the source is considerably more variable than the average quasar. This is promising for both improving the accuracy of the time delays and for using reverberation mapping techniques as an additional probe of the source structure.

A comprehensive description of the results is presented in Fohlmeister et al. 2007 and Fohlmeister et al. 2008.

\section{References}

[1] de Vries, W. H., Becker, R. H., White, R. L., Loomis, C. 2005, AJ, 129, 615

[2] Fohlmeister, J. et al. 2007, ApJ, 662, 62

[3] Fohlmeister, J., Kochanek, C. S., Falco, E. E., Morgan, C. W., Wambsganss 2008, ApJ, 676, 761

[4] Hawkins, M. R. S. 2007, A\&A, 462, 581

[5] Inada, N. et al. 2003, Nature, 426, 810

[6] Inada, N. et al. 2005, PASJ, 57, L7

[7] awaguchi, T., Mineshige, S., Umemura, M., \& Turner, E.L. 1998, ApJ, 504, 671

[8] Kawano, Y., \& Oguri, M. 2006, PASJ, 58, 271

[9] Kochanek, C. S. 2004, ApJ, 605, 58

[10] Kochanek, C. S., Morgan, N. D., Falco, E. E., McLeod, B. A., Winn, J. N., Dembicky, J., \& Ketzeback, B. 2006, ApJ, 640, 47

[11] Kundic, T. et al. 1997, ApJ, 482, 75

[12] Oguri, M., et al. 2004, ApJ, 605, 78

[13] Oguri, M. 2007, ApJ, 660, 1

[14] Pelt, J., Hoff, W., Kayser, R., Refsdal, S., \& Schramm, T. 1994, A\&A, 286,775

[15] Pelt, J., Kayser, R., Refsdal, S., \& Schramm, T. 1996, A\&A, 305, 97

[16] Schild, R.E., \& Thomson, D.J., 1995, Aj, 109, 1070

[17] Sharon, K. et al. 2005, ApJ, 629, 73

[18] Vanden Berk, D.E., et al., 2004, ApJ, 601, 692

[19] Wambsganss, J., 2003, Nature, 426, 781

[20] Wilhite, B.C., Brunner, R.J., Grier, C.J., Schneider, D.P., Vanden Berk, D.E. 2008, MNRAS, 383, 1232

[21] Williams, L. L. R. \& Saha, P. 2004 , AJ, 128, 2631 